\title{
Aspectos epidemiológicos, clínicos e hematológicos de 251 cães portadores de mórula de Ehrlichia spp. naturalmente infectados
}

\author{
[Epidemiological, clinical, and hematological aspects of 251 dogs \\ naturally infected with Ehrlichia spp. morulae] \\ S. Borin ${ }^{1}$, L.Z. Crivelenti ${ }^{2}$, F.A. Ferreira ${ }^{3}$ \\ ${ }^{1}$ Aluna de pós-graduação - FCAV-UNESP - Jaboticabal, SP \\ ${ }^{2}$ Médico veterinário residente - UNIFRAN - Franca, SP \\ ${ }^{3}$ Universidade Federal de Uberlândia - Uberlândia, MG
}

\begin{abstract}
RESUMO
Realizou-se um estudo retrospectivo dos aspectos epidemiológicos, sinais clínicos, dados de exame físico e alterações hematológicas da erliquiose em 251 cães naturalmente infectados por Ehrlichia spp. Dos 4407 casos atendidos em hospital veterinário no período de janeiro de 2002 a dezembro de 2003, verificou-se que 251 cães eram portadores de mórula de Ehrlichia spp. em leucócitos de sangue periférico. Destes, 48 foram eliminados das avaliações por apresentarem patologias concomitantes. Nos 203 cães restantes, verificou-se que houve maior ocorrência em fêmeas $(61,1 \%)$ e que a doença mantevese constante durante todo o período avaliado. Observou-se que 38\% encontravam-se na faixa etária entre um e 23 meses e 58,6\% eram de raça definida. As principais alterações clínicas observadas foram apatia, anorexia/hiporexia, vômito, secreção oculonasal e esplenomegalia. Cento e cinco cães apresentaram temperatura retal entre 38 e $39,5^{\circ} \mathrm{C}$. As alterações observadas com maior frequência no hemograma foram anemia, predominando o tipo normocítica normocrômica $(58,2 \%)$; desvio nuclear de neutrófilos para a esquerda $(67 \%)$ e eosinopenia $(58,1 \%)$.
\end{abstract}

Palavras-chave: cão, erliquiose, Ehrlichia spp., mórula

\begin{abstract}
A study of epidemiological and clinical aspects, alterations of physical exams, and hematological changes of canine ehrlichiosis was performed. A retrospective study was performed in 4,407 dogs referred to a Veterinary Hospital from January 2002 to December 2003. Of all cases, 251 dogs showed Ehrlichia spp. morulae. Among these, 48 were excluded from the study due to other co-infection by other pathologies. In the other 203 evaluated dogs, females (61.1\%) were more infected than males. The dogs aged from one to 23 months (68.6\%) and 58.6\% were definite breed. Emesis, apathy, anorexia/hypoxeria, spleenomegaly, and nasal discharge were the most common signs presented. Rectal temperature was $38-$ $39.5^{\circ} \mathrm{C}$ in 105 dogs. The most usual changes seen during the hematological tests were normochromic and normocitic anemia (58.2\%), a left shift of the neuthrophils (67\%), and eosinopenia (58.1\%).
\end{abstract}

Keywords: dog, ehrlichiosis, Ehrlichia spp., morulae

\section{INTRODUÇ̃̃O}

A erliquiose, considerada uma das mais importantes doenças que acometem os cães (Woody e Hoskins, 1991), é uma infecção potencialmente fatal, tanto para os cães domésticos, quanto para os demais membros da família Canidae (Pyle, 1980; Waner e Harrus, 2000). Trata-se de uma doença muito frequente no Brasil (Labarthe et al., 2003), porém a escassa literatura nacional faz com que os estudos sejam baseados em dados de outros países, onde fatores climáticos, ambientais e sociais diferem das condições brasileiras (Waldemarin et al., 2003).

Recebido em 25 de setembro de 2008 
Os agentes causadores da erliquiose canina pertencem à família Rickettsiaceae (Dagnone et al., 2003). São microrganismos pleomórfos que se caracterizam por serem parasitas intracelulares obrigatórios, tanto no hospedeiro vertebrado, quanto no vetor invertebrado (Woody e Hoskins, 1991). As espécies que parasitam os cães são Ehrlichia equi, E. platy, E. ewing, E. chaffensis (Rikihisa et al., 1991) e E. canis, sendo esta última a mais frequente (Dagnone et al., 2003). Têm como vetor e frequente reservatório o carrapato Rhipicephalus sanguineus, embora outras duas espécies de carrapatos, Amblyomma americanum e Octobius magnini, sejam descritas como potenciais vetores (Rikihisa et al., 1991). A transmissão ocorre também por meio de transfusões sanguíneas de um cão infectado para outro susceptível (López et al., 1999).

Do ponto de vista clínico, a erliquiose manifestase de forma aguda, subclínica ou crônica (Woody e Hoskins, 1991). Acomete cães de idades variadas e já foi relatada em cães com dois meses a 13 anos de idade (Kuehn e Gaunt, 1985). A suspeita clínica pode ser confirmada por meio do achado de mórulas e inclusões de Ehrlichia spp. em leucócitos de esfregaços sanguíneos (Andereg e Passos, 1999; Oriá et al., 2004). No entanto, é importante ressaltar que a ausência de parasitas em esfregaços de sangue periférico não exclui a possibilidade de infecção (Woody e Hoskins, 1991).

Dentre as alterações hematológicas relatadas com maior frequência na literatura consultada, destacam-se a anemia arregenerativa (Kuehn e Gaunt, 1985; Woody e Hoskins, 1991; Waner e Harrus, 2000; Oriá, 2001; Moreira et al., 2003; Waldemarin et al., 2003; Oriá et al., 2004) e, em menor frequência, a anemia regenerativa (Woody e Hoskins, 1991).

Das alterações presentes no leucograma, destacam-se o desvio nuclear de neutrófilos para a esquerda e a eosinopenia (Waddle e Littman, 1988; Moreira et al., 2003; Waldemarin et al., 2003). Outros achados como leucopenia (Kuehn e Gaunt, 1985; Waddle e Littman, 1988; Waner e Harrus, 2000; Waldemarin et al., 2003) e monocitopenia (Waddle e Littman, 1988) são considerados menos frequentes.

Este trabalho teve como objetivo realizar um estudo retrospectivo de casos de erliquiose em cães naturalmente infectados, atendidos em hospital veterinário.

\section{MATERIAL E MÉTODOS}

Este estudo baseou-se na avaliação da casuística natural de atendimentos de cães do Hospital Veterinário da Universidade Federal de Uberlândia, em Uberlândia, MG, entre janeiro de 2002 e dezembro de 2003. Fez-se o levantamento de todos os casos clínicos dos animais atendidos neste período, discriminando-os em machos e fêmeas. Foram levadas em consideração as fichas clínicas e laboratoriais dos cães que apresentavam mórula de Ehrlichia spp. em leucócitos de esfregaços sanguíneos, confeccionados com sangue obtido por punção de vasos marginais do pavilhão auricular e corados pelo May-Grünwald-Giemsa (FerreiraNeto et al., 1982).

Dos 251 cães positivos, obtiveram-se os seguintes dados: idade, sexo, raça e data do atendimento, sinais clínicos e alterações observadas ao exame físico (temperatura corporal, infecções concomitantes, contato com carrapatos) e quadro hematológico (eritrograma e leucograma).

Realizou-se uma análise descritiva e aplicou-se o teste de qui-quadrado (Siegel, 1975) aos parâmetros de frequência e porcentagens, estabelecendo-se nível de significância em 0,05, em uma prova bicaudal.

\section{RESULTADOS E DISCUSSÃO}

No período de janeiro de 2002 a dezembro de 2003, foram atendidos 4407 cães, sendo 2062 machos $(46,8 \%)$ e 2345 fêmeas $(53,2 \%)$. Desse total, foram encontrados 251 portadores de mórula de Ehrlichia spp.

Quarenta e oito animais (19,1\%) com erliquiose apresentaram patologias concomitantes, dentre estas associações com outros hemoparasitas (12,7\%), destacando-se Babesia spp. e Hepatozoon spp. Coinfecções com hemoparasitas já foram verificadas em $10 \%$ dos cães com erliquiose em Belo Horizonte, associadas à presença de Babesia canis e Mycoplasma haemocanis (Moreira et al., 2003), e dentre os 5,9\% de cães errantes da região de Botucatu, SP infectados por Hepatozoon canis, 
3,2\% apresentaram-se coinfectados por E. canis e B. canis (O’Dwyer et al., 2006).

Dos 251 cães positivos à pesquisa de hemoparasitas em esfregaço sanguíneo, 203 (80,9\%) apresentaram exclusivamente Ehrlichia spp. Considerando que a visualização microscópica de mórula de Ehrlichia spp. no interior de leucócitos ocorre durante a fase aguda da doença e em aproximadamente $4 \%$ dos casos (Waner e Harrus, 2000), este quadro sugere alta porcentagem de casos de erliquiose atendidos, pois o encontro na fase subclínica ou crônica de mórula intracitoplasmática é raro.

A deteç̧ão de mórula de E. canis é mais eficiente em esfregaço de sangue periférico (Castro, 1997). Supõe-se que a rotineira realização de exames para pesquisa de hemoparasitas, procedidos a partir de esfregaços confeccionados com sangue obtido por punção de vasos marginais do pavilhão auricular dos cães, contribuiu para o achado de grande quantidade de mórulas intracitoplasmáticas.

Dos 203 cães, 79 eram machos (38,9\%) e 124, fêmeas $(61,1 \%)$, isto é, $5,3 \%$ do total de fêmeas e $3,8 \%$ do total de machos atendidos eram portadores de mórula de Ehrlichia spp. A ocorrência da erliquiose canina em fêmeas foi significativamente mais alta. Este resultado confirma os já obtidos na literatura nacional, em que $61,5 \%$ dos cães acometidos pela doença eram fêmeas (Moreira et al., 2003).

A casuística da erliquiose manteve-se estável no período avaliado, talvez devido ao difícil controle e ao clima da região favorável ao desenvolvimento de ectoparasitas (Fig. 1).

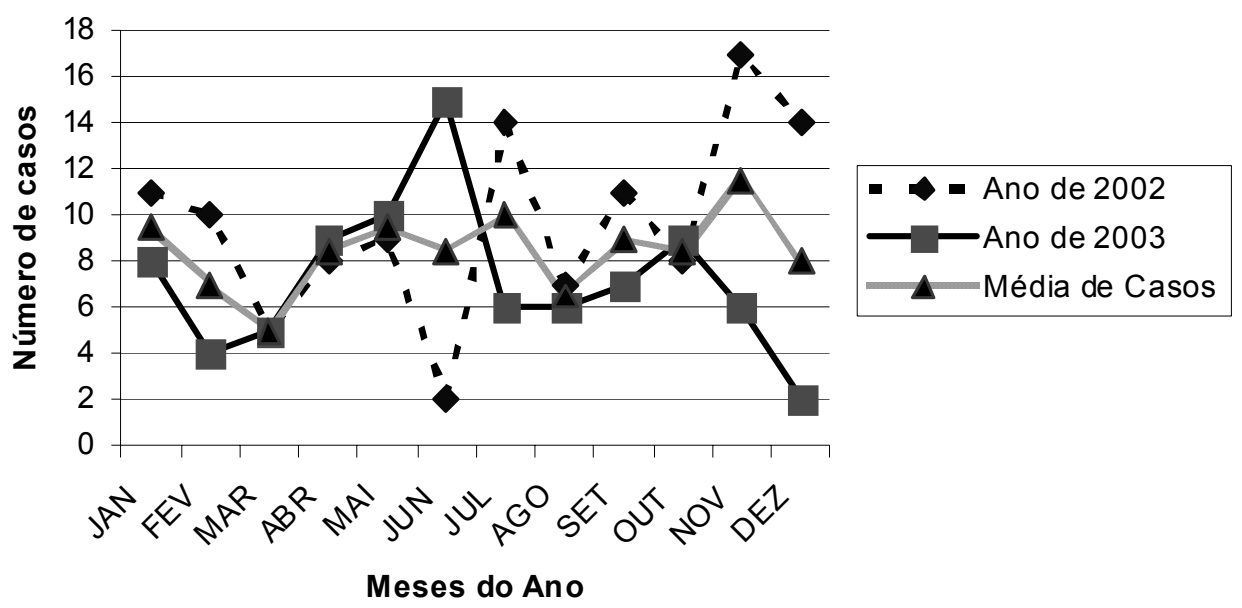

Figura 1. Distribuição mensal dos casos de cães infectados por Ehrlichia spp. em Uberlândia, MG, nos anos de 2002 e 2003.

Dos 203 cães, 119 (59\%) eram de raças definidas, destacando-se a Poodle (20\%), a Pastor Alemão (13\%) e a Pinscher (13\%) como as raças mais frequentes. Em análises semelhantes, autores sugeriram a raça Pastor Alemão como a mais susceptível (Elias, 1991; Rikihisa et al., 1991). No entanto nada se pode afirmar em relação à incidência, visto não se ter a dimensão exata da casuística dessas raças.

Foram observados cães entre um mês e 18 anos infectados por Ehrlichia spp. Destes, 38\% encontravam-se na faixa etária de um a 23 meses de idade, valores semelhantes aos descritos por Moreira et al. (2003).

Os principais sinais clínicos e alterações observados ao exame físico nos cães portadores de mórulas de Ehrlichia spp. (Fig. 2) foram semelhantes aos da literatura consultada (Moreira et al., 2003; Oriá et al., 2004). Os outros sinais com menor ocorrência foram lesão ocular $(8,4 \%)$, linfadenomegalia $(6,9 \%)$, claudicação $(6,4 \%)$, ascite $(3 \%)$ e petéquias $(1 \%)$. 


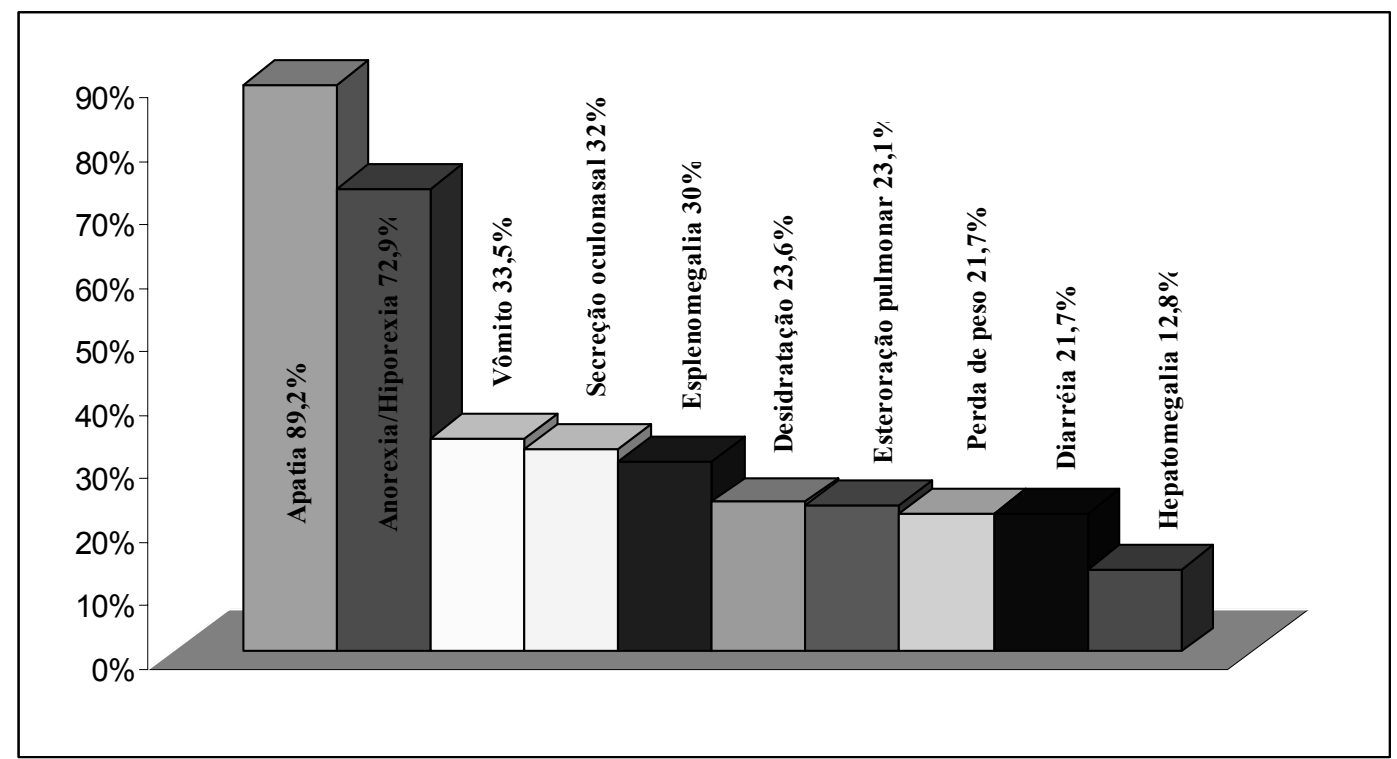

Figura 2. Principais sinais clínicos e alterações ao exame físico dos cães portadores de mórula de Ehrlichia spp.

Dos 203, 77 cães $(38 \%)$ apresentaram temperatura retal igual ou superior a $39,6^{\circ} \mathrm{C}, 105$ $(52 \%)$ entre 38 e $39,5^{\circ} \mathrm{C}$, e $21(10 \%)$ temperatura inferior a $37,9^{\circ} \mathrm{C}$. Tais dados diferem dos descritos por Moreira et al. (2003), em cujo estudo todos os cães apresentaram temperatura superior a $39,7 \%$.

Um dado importante a ser ressaltado é o de que a maioria dos proprietários (53\%) informou que não houve contato dos cães com carrapatos.

Cento e sessenta e sete cães (82,3\%) apresentaram anemia, a mais frequente alteração ocorrida no eritrograma, já citada por Woody e Hoskins (1991), Oriá (2001), Moreira et al. (2003), Waldemarin et al. (2003) e Oriá et al. (2004). De acordo com os valores do volume corpuscular médio (VCM) e da concentração de hemoglobina corpuscular média (CHCM) dos cães que apresentaram anemia, 118 animais $(58,2 \%)$ apresentaram anemia do tipo normocítica normocrômica. Atuação do sistema monocítico-fagocitário, lise celular pela ação do sistema complemento e supressão da eritropoiese na medula óssea podem ser os mecanismos apontados como responsáveis pelo quadro anêmico da doença (Moreira et al., 2003), sugerindo resposta medular não regenerativa ou pouco responsiva (Waldemarin et al., 2003; Bush, 2004). É importante ressaltar que 36
$(17,7 \%)$ cães não apresentaram alterações relativas à contagem eritrocitária e $21(10,3 \%)$, anemia normocítica hipocrômica. Os demais pacientes apresentaram outros tipos de anemia, menos frequentes.

Os dados do leucograma encontram-se na Tab. 1. A contagem leucocitária normal encontrada é condizente com os achados de Waldemarin et al. (2003). A leucopenia verificada é semelhante à observada por Moreira et al. (2003) e mais baixa que os $65,9 \%$ e $32,7 \%$ encontrados por Oriá (2001) e Waldemarin et al. (2003), respectivamente.

Quanto à contagem diferencial de leucócitos, o resultado mais expressivo foi o desvio nuclear de neutrófilos para a esquerda, observado em 136 animais (67\%), valor semelhante aos $66,7 \%$ citados por Moreira et al. (2003) e maior que os $37,2 \%$ encontrados por Oriá (2001). Cento e dezoito cães apresentaram eosinopenia $(58,1 \%)$, assim como observado por Kuehn e Gaunt (1985), Waddle e Littman (1988), Moreira et al. (2003) e Waldemarin et al. (2003), provavelmente devido à ação dos glicocorticoides endógenos, liberados sob situação de estresse e em quadros de infecção grave, reduzindo a produção de eosinófilos pela medula óssea e ocasionando aumento da sua destruição (Waldemarin et al., 2003; Bush, 2004). 
Tabela 1. Ocorrência de achados no de leucograma em 203 cães naturalmente infectados por Ehrlichia spp., atendidos no período de janeiro de 2002 a dezembro de 2003

\begin{tabular}{lccccccc}
\multirow{2}{*}{ Característica } & \multicolumn{2}{c}{ Normal } & \multicolumn{2}{c}{ Elevado } & \multicolumn{2}{c}{ Diminuído } & \multirow{2}{*}{ Total } \\
\cline { 2 - 6 } & $\mathrm{N}^{\circ}$ & $\%$ & $\mathrm{~N}^{\circ}$ & $\%$ & $\mathrm{~N}^{\circ}$ & $\%$ & \\
\hline Leucócitos & 112 & 55,17 & 41 & 20,19 & 50 & 24,63 & 203 \\
Neutrófilos totais & 126 & 62,07 & 38 & 18,72 & 39 & 19,21 & 203 \\
Linfócitos & 179 & 88,18 & 03 & 1,48 & 21 & 10,34 & 203 \\
Eosinófilos & 80 & 39,41 & 05 & 2,46 & 118 & 58,13 & 203 \\
Monócitos & 165 & 81,28 & 12 & 5,91 & 26 & 12,81 & 203 \\
Basófilos & 199 & 98,03 & 04 & 1,97 & - & 00,00 & 203 \\
\hline
\end{tabular}

\section{CONCLUSÃO}

Sugere-se a confecção de esfregaços de sangue periférico como rotina na clínica de pequenos animais, por se tratar de um exame rápido, de baixo custo e com resultados precisos no diagnóstico da doença, quando positivo.

\section{AGRADECIMENTOS}

Aos residentes, docentes e funcionários do Hospital Veterinário da Universidade Federal de Uberlândia, MG. À médica veterinária Mariana Cristina Hoeppner Rondelli, pela tradução do resumo.

\section{REFERÊNCIAS BIBLIOGRÁFICAS}

ANDEREG, P.I.; PASSOS, L.M.F. Erliquiose canina: revisão. Rev. Clin. Vet., v.4, p.31-38, 1999.

BUSH, B.M. Interpretação de resultados laboratoriais para clínicos de pequenos animais. São Paulo: Roca, 2004. 376p.

CASTRO, M.B. Alterações clínicas, anatomopatológicas e imunopatológicas na erliquiose aguda experimental em cães. 1997. 97f. Dissertação (Mestrado) - Faculdade de Ciências Agrárias e Veterinárias, Universidade Estadual Paulista, Jaboticabal, SP.

DAGNONE, A.S.; MORAIS, H.S.A.; VIDOTTO, M.C. et al. Ehrlichiosis in anemic, thrombocytopenic, or tick-infested dogs from a Hospital population in South Brazil. Vet. Parasitol., v.117, p.285-290, 2003.

ELIAS, E. Diagnosis of ehrlichiosis from the presence of inclusion bodies or morulae of $E$. canis. J. Small Anim. Pract., v.33, p.540-543, 1991.
FERREIRA NETO, J.M.; VIANA, E.S.; MAGALHÃES, L.M. Patologia clínica veterinária. Belo Horizonte: Rabelo, 1982. 279p.

KUEHN, N.F.; GAUNT, S.D. Clinical and hematologic findings in canine ehrlichiosis. $J$. Am. Vet. Med. Assoc., v.186, p.355-358, 1985.

LABARTHE, N.; CAMPOS PEREIRA, M.; BARRARINI, O. et al. Serologic prevalence of Dirofilaria immitis, Ehrlichia canis, and Borrelia burgdorferi infections in Brazil. Vet. Ther., v.4, p.67-75, 2003.

LÓPEZ, J.; CASTILLO, A.; MUNOZ, M. et al. Hallazgo de Ehrlichia canis en Chile, informe preliminar. Arch. Med. Vet., v.31, p.211-214, 1999.

MOREIRA, S.M.; BASTOS, C.V.; ARAÚJO, R.B. et al. Estudo retrospectivo (1998 a 2001) da erliquiose canina em Belo Horizonte. Arq. Bras. Med. Vet. Zootec., v.55, p.141-147, 2003.

O'DWYER, L.H., SAITO, M.E., HASEGAWA, M.Y. et al. Prevalence, hematology and serum biochemistry in stray dogs naturally infected by Hepatozoon canis in São Paulo. Arq. Bras. Med. Vet. Zootec., v.58, p.688-690, 2006.

ORIÁ, A.P. Correlação entre uveítes, achados de patologia clínica, sorológicos (Reação de imunofluorescência indireta e Dot-blot ELISA) e de anatomopatologia do bulbo do olho, em animais da espécie canina, natural $e$ experimentalmente infectados por Ehrlichia canis. 2001. 69f. Dissertação (Mestrado) Faculdade de Ciências Agrárias e Veterinárias, Universidade Estadual Paulista, Jaboticabal, SP.

ORIÁ, A.P.; PEREIRA, P.M.; LAUS, J.L. Uveíte em cães infectados com Ehrlichia canis. Cienc. Rural, v.34, p.1289-1295, 2004.

PYLE, R. L. Canine ehrlichiosis. J. Am. Vet. Med. Assoc., v.177, p.1197-1199, 1980. 
RIKIHISA,Y.; EWING, S.A.; FOX, J.C et al. Analyses of Ehrlichia canis and a canine granulocitic Ehrlichia infection. J. Clin. Microbiol., v.30, p.143-148, 1991.

SIEGEL, S. Estatística não-paramétrica, para as ciências do comportamento. São Paulo: McGraw-Hill do Brasil, 1975. 350p.

WADDLE, J.R.; LITTMAN. M.P. A retrospective study of 27 cases of naturally occurring canine ehrlichiosis. J. Am. Anim. Hosp. Assoc., v.24, p.615-620, 1988.
WALDEMARIN, K.C.A.; MUNDIM, A.V.; BASTOS, J.E.D. et al. Alterações hematológicas em cães (Canis familiaris) naturalmente infectados por Ehrlichia spp. Vet. Not., v.9, p.2329, 2003.

WANER, T.; HARRUS, S. Canine monocytic ehrlichiosis. In: __ Recent advances in canine infectious diseases. Ithaca: L.E. Carmachael, 2000.

WOODY, B.J.; HOSKINS, J.D. Ehrlichial diseases of dogs. Vet. Clin. N. Am.: Small Anim. Pract., v.21, p.75-98, 1991. 